\title{
A Look into Obstructive Sleep Apnea among Ethiopians
}

\author{
Asefa Mekonnen ${ }^{1}$, Monika Gasiorek ${ }^{2}$, Marc Key ${ }^{1}$, Gregory Stimac ${ }^{2 *}$
}

\section{OPEN ACCESS}

Citation: Asefa Mekonnen, Monika Gasiorek, Marc Key, Gregory Stimac. A Look into Obstructive Sleep Apnea among Ethiopians. Ethiop J Health Sci.2019;29(5):585.doi:http://dx.doi.org/ 10.4314/ejhs.v29i5.8

Received: April 30, 2019

Accepted: July 22, 2019

Published: September 1, 2019

Copyright: (C) 2019 Asefa Mekonnen, et

al. This is an open access article distributed under the terms of the Creative Commons Attribution License, which permits unrestricted use, distribution, and reproduction in any medium, provided the original author and source are credited.

Funding: Nil

Competing Interests: The authors declare that this manuscript was approved by all authors in its form and that no competing interest exists.

Affiliation and Correspondence:

${ }^{1}$ Sleep Medicine, Rockville Internal Medicine Group, Rockville,

Maryland, United States

${ }^{2}$ School of Medicine, Georgetown

University, Washington, District of

Columbia, United States

*Email: gps34@georgetown.edu

\section{ABSTRACT}

BACKGROUND: Obstructive Sleep Apnea (OSA) is prevalent throughout the world. However, there are currently limited data concerning the prevalence of OSA in populations that originate from developing countries; the prevalence of OSA is expected to rise in these countries. OSA is poorly characterized amongst Ethiopians, and our study is the first to describe clinical characteristics of OSA among Ethiopians.

METHODS: We conducted a retrospective study of primarily Ethiopian patients at an internal medicine clinic in Rockville, Maryland. All patients $(n=24)$ were evaluated for daytime sleepiness using the Epworth Sleepiness Scale (ESS) and received physical examinations and polysomnograms (PSG) by either portable monitoring (Itamar WatchPAT 200 device) or in-lab. Statistical analyses were performed in $R$.

RESULTS: Linear regression model of Body-Mass Index (BMI) and Apnea-Hypopnea Index (AHI) indicated that for every 1-unit increase in BMI, there was a 0.8657-unit increase in AHI $(p<0.05)$. Pearson's correlation coefficient indicateda positive linear relationship between BMI and AHI (0.47) $(p<0.05)$. Adjusted linear regression model for $A H I$ and oxygen saturation indicated that for every 1-unit increase of AHI, there was a 0.8452-unit decrease in nocturnal oxygen saturation $(p<0.05)$. Pearson's correlation coefficient did not demonstrate significance between AHI and oxygen desaturation $(p=0.062)$. Patients received either continuous positive airway pressure $(\mathrm{CPAP})(\mathrm{n}=15)$ or oral appliance therapy $(n=3)$.

CONCLUSION: All patients who complied with therapy reported improved sleep quality, snoring resolution, and improved daytime alertness. Practitioners in developing countries should suspect OSA in the right clinical setting and offer diagnostic and therapeutic services when available.

KEYWORDS: Apnea; Sleep Apnea, Obstructive; Ethiopia; Continuous Positive Airway Pressure; Sleep 


\section{INTRODUCTION}

Obstructive Sleep Apnea (OSA) is a highly prevalent disorder with known adverse cardiometabolic consequences (1). Although OSA affects people in all parts of the world, there is little data on the state of the disorder in developing countries, particularly those in Africa. Appropriate diagnosis and management of OSA requires a trained and informed healthcare professional with access to diagnostic and therapeutic technology. Currently, the delivery of sleep health services is non-existent in many developing countries. The prevalence of OSA and its negative effects is expected to rise as society continues to change with urbanization, changes in lifestyle, and the rise of obesity worldwide. The effect of untreated OSA on an individual significantly impacts quality of life and society at large. It is urgent that medical professionals and the public in developing nations are informed about the disorder, its risk factors, and the diagnostic and therapeutic standards.

In the last few years, many patients traveled from different parts of Africa for sleep medicine consultation services at a premier sleep disorders center in Rockville, Maryland, with people of Ethiopian descent representing the majority. This study is the first to describe clinical and polysomnographic data in this special population. The objective of this article is to share our experience and raise awareness regarding OSA for practitioners in the developing world.

\section{MATERIALS AND METHODS}

We reviewed demographic, clinical, and laboratory data for Ethiopian patients who sought a sleep medicine consultation for possible sleep disorders of breathing between January 2014 and July 2017 at a premier sleep disorders center in Rockville, Maryland. Electronic medical records of the Rockville Internal Medicine Group and Premier Sleep Disorders Center were our sources of clinical and polysomnographic data. All patients had detailed sleep history and evaluation of daytime sleepiness by the Epworth sleepiness scale (ESS), a detailed physical examination, and a polysomnographic study. Diagnostic and therapeutic sleep studies were done either by portable monitoring using the Itamar WatchPAT
200 sleep diagnostic device or an in-lab polysomnographic study.

Setting: Premier Sleep Disorders Center is an American Academy of Sleep Medicine accredited comprehensive sleep medicine center in Rockville, Maryland. All patients were evaluated and followed by a board certified sleep medicine physician. Pre- and post-diagnostic visits were made to discuss test results and therapeutic options. Polysomnographic data was scored and reviewed according to the standard American Academy of Sleep Medicine diagnostic criteria by a board certified sleep technologist and a board certified sleep medicine physician. The diagnosis of OSA was made using an apnea and hypopnea index of 5 and above in the setting of either daytime hypersomnia or other comorbidity (2). Continuous Positive Airway Pressure (CPAP) and Oral Appliance (OA) therapy were the main therapeutic interventions in addition to sleep hygiene and weight loss measures. Patients who failed CPAP therapy were treated with oral appliance. All patients had follow-up visits of 1-6 weeks after initiation of therapy depending on their travel status.

Statistical analysis: Statistical analyses of the association between Body-Mass Index (BMI) and the OSA Apnea-Hypopnea Index (AHI), as well as between AHI and nocturnal oxygen desaturation, were performed with the $\mathrm{R}$ package, version 3.4.2. Linear regression model was fitted for both sets of continuous variables and Pearson's correlation coefficient was calculated for each relationship. OSA was modeled both as a continuous variable (AHI) and as an ordered categorical variable (apnea severity). Apnea severity levels were defined as none (AHI $=1-4)$, mild (AHI $=5-15)$, moderate $(\mathrm{AHI}=16-30)$, and severe $(\mathrm{AHI}>30)$.

\section{RESULTS}

A total of 24 patients received sleep medicine consultation services for a suspected sleep disorder of breathing. Descriptive characteristics of the sample population are shown in Table 1. The group was predominantly male $(79.1 \%)$ with an average age of 53.9 (range: 32-76) and an average BMI of 29.5 (range: 18.6-40.1). According to CDC standards for BMI classification, $41.7 \%$ of the subjects were 
overweight and another $41.7 \%$ were obese or morbidly obese (3). While all patients were of Ethiopian descent, the majority $(54.2 \%)$ traveled from Ethiopia seeking medical care due to a paucity of sleep medicine services in Ethiopia. The rest $(45.8 \%)$ were permanent residents of the United States.

All patients underwent a sleep study either by portable sleep monitoring $(70.8 \%)$ or in-lab polysomnography $(29.2 \%)$ for diagnosis; 21

Table 1: Patient demographics

\begin{tabular}{|c|c|c|}
\hline Characteristic & Category & Number (percent) \\
\hline \multirow[t]{2}{*}{ Gender } & Male & $19(79.1)$ \\
\hline & Female & $5(20.8)$ \\
\hline \multirow[t]{2}{*}{ Age } & Mean $(25 \%-75 \%)$ & $53.9(46.5-62.5)$ \\
\hline & Range & $32-76$ \\
\hline \multirow[t]{2}{*}{ Country of Residence } & Ethiopia & $13(54.2)$ \\
\hline & United States & $11(45.8)$ \\
\hline \multirow[t]{2}{*}{ BMI $\left(\mathrm{kg} / \mathrm{m}^{2}\right)$} & Mean $(25 \%-75 \%)$ & $29.5(26.1-33.3)$ \\
\hline & Range & $18.6-40.1$ \\
\hline \multirow[t]{6}{*}{ Symptoms } & Snoring & $23(95.8)$ \\
\hline & Un-refreshing sleep & $24(100)$ \\
\hline & Witnessed apnea & $14(58.3)$ \\
\hline & Fatigue & $23(95.8)$ \\
\hline & Headache & $8(33.3)$ \\
\hline & $\mathrm{ESS}>8$ & $19(79.1)$ \\
\hline \multirow[t]{2}{*}{ Comorbidities } & Diabetes mellitus & $6(25)$ \\
\hline & Hypertension & $13(54.2)$ \\
\hline \multirow[t]{2}{*}{ Diagnostic evaluation } & PSG & $7(29.2)$ \\
\hline & Home study & $17(70.8)$ \\
\hline \multirow[t]{4}{*}{ Degree of Apnea } & None & $3(12.5)$ \\
\hline & Mild & $12(50)$ \\
\hline & Moderate & $6(25)$ \\
\hline & Severe & $3(12.5)$ \\
\hline
\end{tabular}

Linear regression model was run to test the association between BMI and AHI as continuous variables (Table 2). The initial model included age and gender as possible covariates, but neither was significant. Tested separately, neither age nor gender showed any suggestion of an association with AHI. The final model included only BMI as an independent variable. The linear regression patients met the diagnostic criteria for OSA with an overall prevalence rate of $87.5 \%$. Presenting symptoms included un-refreshing sleep (100\%), fatigue $(95.8 \%)$, snoring $(95.8 \%)$, daytime hypersomnia given an ESS $>8(79.1 \%)$, witnessed apnea $(58.3 \%)$, and headache $(33.3 \%)$. Among the 21 patients with OSA defined by an AHI of 5 or greater, only $9(42.9 \%)$ had a BMI greater than 30 .

model demonstrated that for every increase of 1 unit in BMI, there was an expected increase of 0.8657 units in AHI (p-value $<0.05$ ). Furthermore, there was a moderate positive linear relationship between BMI and AHI as quantified by Pearson's correlation coefficient of 0.47 (pvalue $<0.05$ ). Although the association was significant, it did not explain a large portion of the variance in AHI, as can be seen in Figure 1A. 
Table 2: Linear regression for BMI and AHI

\begin{tabular}{lc}
\hline & Dependent variable \\
\hline O2 Sat & AHI \\
& $0.866^{* *}$ \\
Constant & $(0.348)$ \\
& $-10.574^{*}$ \\
Observations & $(10.438)$ \\
$\mathrm{R}^{2}$ & 24 \\
Adjusted $\mathrm{R}^{2}$ & 0.220 \\
Residual Std. Error & 0.184 \\
F Statistic & $9.231(\mathrm{df}=22)$ \\
Note & $6.199^{* *}(\mathrm{df}=1 ; 22)$ \\
& $p<0.05$
\end{tabular}
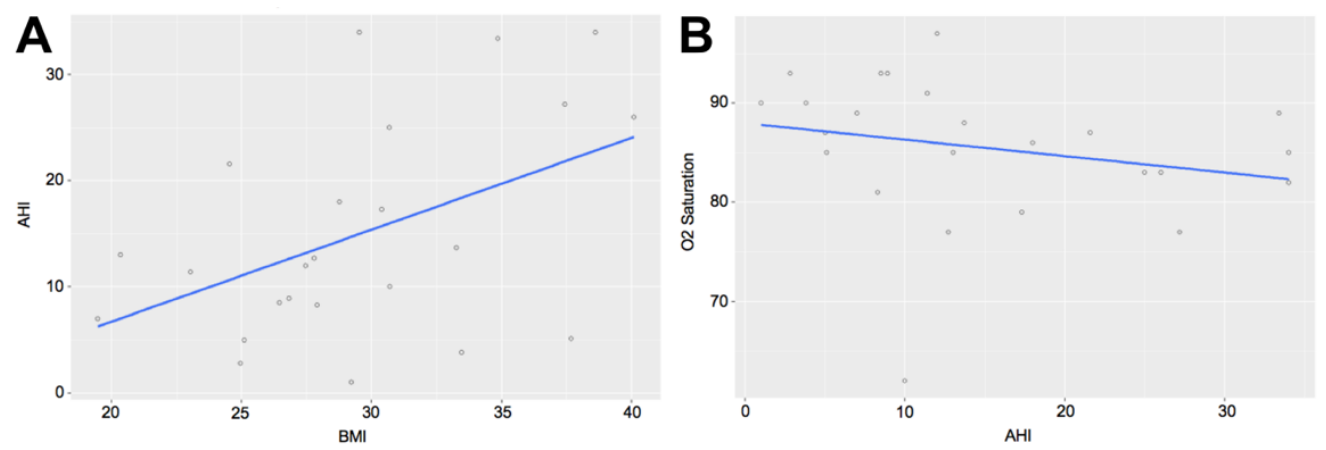

Figure 1A: Scatterplot with trendline for apnea-hypopnea index (AHI) versus body-mass index (BMI);

Figure 1B: Scatterplot with trendline for $\mathrm{O}_{2}$ saturation versus apnea-hypopnea index (AHI)

A separate linear regression model was run to test the association between AHI and oxygen saturation level as continuous variables. Initially there was not a significant association between oxygen saturation and AHI (Table 3, p-value > 0.05). However, excluding a single outlier for whom the data demonstrated a maximum desaturation to $62 \%$, an adjusted linear regression showed that for every increase in one unit of AHI, there was a 0.8452 unit decrease in nocturnal oxygen saturation (Table 4 , p-value $<0.05$ ). The Pearson's correlation coefficient for 23 subjects, excluding the aforementioned outlier, demonstrated an almost significant moderate negative linear association between $\mathrm{AHI}$ and oxygen desaturation $(\mathrm{p}$-value $=0.062$ ). Although the trend was in the right direction, that is, the estimate was in the negative direction, again, the actual values of AHI varied greatly from the model's predicted values (Figure 1B). Classification of sleep apnea by severity did not yield any significant associations between severity and either BMI or oxygen saturation (analyses not shown).

Table 3: Linear Regression for $\mathrm{O}_{2}$ saturation and AHI with outliers

\begin{tabular}{lc}
\hline & Dependent variable \\
\hline & AHI \\
\hline O2 Sat & -0.334 \\
& $(0.294)$ \\
Constant & $43.546^{*}$ \\
& $(25.240)$ \\
Observations & 24 \\
$\mathrm{R}^{2}$ & 0.055 \\
Adjusted $\mathrm{R}^{2}$ & 0.012 \\
Residual Std. Error & $10.157(\mathrm{df}=22)$ \\
F Statistic & $1.289(\mathrm{df}=1 ; 22)$ \\
Note & $p<0.05$ \\
\hline
\end{tabular}

DOI: http://dx.doi.org/10.4314/ejhs.v29i5.8 
CPAP was elected as a first line therapy in 15 patients, while oral appliance therapy was used as first line in 3 patients. Among patients who were elected for CPAP, 4 failed CPAP and 3 of those patients followed with oral appliance therapy. Three patients declined therapy.

CPAP therapy was initiated after in-lab polysomnographic pap titration or using auto CPAP. FDA-approved prefabricated or custom oral appliances were used when considering oral appliance therapy. As most patients were paying out of pocket, economic factors affected the decisions made for both diagnostic and therapeutic methods of intervention.

At follow-up, all patients who complied with either CPAP or oral appliance therapy reported improved sleep quality, resolution of snoring, and improved daytime alertness. Long-term follow-up was not possible as the majority of the patients resided in Ethiopia. The longest follow-up was done for patients residing in the United States. The overall success of therapy was impressive.

Table 4: Linear Regression for $\mathrm{O}_{2}$ saturation and AHI without outliers.

\begin{tabular}{lc}
\hline & Dependent variable \\
\hline O2 Sat & AHI \\
& $-0.845^{* *}$ \\
Constant & $(0.387)$ \\
& $88.337 *$ \\
& $(33.547)$ \\
\hline Observations & 23 \\
R $^{2}$ & 0.185 \\
Adjusted R & 0.146 \\
Residual Std. Error & $9.604(\mathrm{df}=21)$ \\
F Statistic & $4.769^{* *}(\mathrm{df}=1 ; 21)$ \\
\hline Note & $p<0.05$ \\
\hline
\end{tabular}

\section{DISCUSSION}

OSA is a highly prevalent and under-diagnosed medical condition. In the developed world, including the United States of America, only $20 \%$ of patients are diagnosed and treated (4). In Africa, with a rapidly growing population of over one billion, the delivery of sleep medicine services is at its embryonic stage. Excluding Southern Africa, few countries in the continent have started incorporation of sleep medicine into the healthcare system. Egypt,
Nigeria, Morocco, and Tunisia are a few African countries that are taking the lead (5-9). Lack of knowledge about sleep and its disorders, lack of trained personnel, the high level of capital needed to establish sleep medicine services, and the unavailability of diagnostic centers and therapeutic devices are believed to be some of the reasons for Africa's lag in incorporating sleep medicine into its patient services. Studies conducted among graduating medical students in Nigeria found that only $41 \%$ of the students were confident in recognizing signs and symptoms suggestive of OSA (10). For the above reasons, the state of OSA in African countries, including its prevalence, clinical presentations, clinical consequences, and interactions with other comorbid medical conditions, is largely unknown.

Nonetheless, a few preliminary studies indicate that it is a highly prevalent medical condition. A study among Nigerian young adults found a $19 \%$ (22\% males, $16 \%$ females) prevalence of clinical OSA (study conducted using a validated questionnaire) (5). In Ethiopia, 19\% of college students were found to have high risk for clinical OSA (11). A study in Egypt found a prevalence of $42 \%$ among children with epilepsy, with an observation that OSA contributed to seizure frequency (6). Nigerian adult hypertensives were found to have a $52 \%$ prevalence of clinical sleep apnea while patients with type II diabetes mellitus had a $27 \%$ prevalence in the same population $(7,8)$. In Morocco, among patients seen for pulmonary consultation at a tertiary medical center, a $56.7 \%$ prevalence of OSA was observed (9). These preliminary studies indicate that the burden of OSA in Africa is high. Additionally, some of the above studies identified obesity (high BMI and large neck size) as risk factors. However, detailed clinical descriptions of subjects, objective diagnostic testing supported by polysomnographic monitoring, severity of disease classification, and management strategies have not been discussed in the few studies published on OSA among Africans.

Ethiopia, Africa's second most populous nation with a population above 90 million in the midst of demographic transformation, has just started implementing diagnostic sleep medicine services. Our study is the first to describe clinical characteristics of OSA among Ethiopians. The confirmation of the diagnosis with objective 
polysomnographic testing is one strength of our observations. We found all spectrums of the disease, including mild, moderate and severe OSA, in our cohort. BMI correlated as a risk factor for increased AHI in our series $(p<0.05)$, although the association explained only a small part of the variance in AHI, as demonstrated in Figure 1A, where actual values of AHI were widely dispersed above and below the model's predicted values. We also found that there was a significant nocturnal oxygen arterial desaturation at the mild and moderate stages of the disease. A linear model testing the association between AHI and oxygen saturation was significant only with the exclusion of a single outlier. Again, as shown in Figure 1B, there was a wide dispersion and variance in values from the predicted value line in the model. Nonetheless, this is a critical observation as craniofacial features may play a significant role in the causation of the diseases among Ethiopians, in addition to obesity. It also suggests that Ethiopians may suffer serious consequences of the disease even in its milder forms, as they exhibit significant intermittent nocturnal hypoxia. This has been suspected among African Americans in the United States.

We understand that due to the small size of the study population and because it represents a highly selected patient population who had access to a specialized service in the United States, the findings from this study may not be directly translatable to the general public of Ethiopia. Despite this limitation, we can conclude that this is the first study reporting on a very important clinical syndrome among Ethiopians that may have far-reaching consequences. The authors call on the attention of practitioners in developing countries to suspect OSA in the right clinical setting and offer a diagnostic and therapeutic service when available.

\section{ACKNOWLEDGEMENTS}

Stephen Durako: Provided substantial contributions to statistical analysis and interpretation of findings.

\section{REFERENCES}

1. Natale E, Pistono M. Obstructive sleep apnea: How to diagnose and treat this ever more common disorder. $J$ Cardiovasc Med. 2017;18(suppl 1):e30-4.
2. Kapur VK, Auckley DH, Chowdhuri S, et al. Clinical practice guideline for diagnostic testing for adult obstructive sleep apnea: An American academy of sleep medicine clinical practice guideline. J Clin Sleep Med. 2017;13(3):479.

3. Defining Adult Overweight and Obesity Overweight \&amp; Obesity | CDC. Available from:

https://www.cdc.gov/obesity/adult/defining.html

4. Sleep Apnea Information for Clinicians. American Sleep Apnea Association. 2017. Available from: https://www.sleepapnea.org/learn/sleep-apneainformation-clinicians/

5. Adewole OO, Hakeem A, Fola A, et al. Obstructive sleep apnea among adults in Nigeria. J Natl Med Assoc. 2009;101(7):720.

6. Urquhart DS, Kehinde OO, Mclellan AE. Observational pilot study of reported symptoms of obstructive sleep apnoea in children with epilepsy. Dev Med Child Neurol. 2016;58(10):1063-8.

7. Akintunde AA, Okunola OO, Oluyombo R, Oladosu YO, Opadijo OG. Snoring and obstructive sleep apnoea syndrome among hypertensive Nigerians: prevalence and clinical correlates. Pan Afr Med J. 2012;11:75.

8. Obaseki D, Obaseki J, Abidoye I, et al. Prevalence and predictors of obstructive sleep apnea syndrome in a sample of patients with type 2 Diabetes Mellitus in Nigeria. Niger Med J. 2014;55(1):24.

9. Jniene A, el Ftouh M, Fihry MT el F. Sleep apnea syndrome: experience of the pulmonology department in Ibn Sina Hospital, Rabat, Morocco. Pan Afr Med J. 2012;13:28.

10. Ozoh OB, Iwuala SO, Desalu OO, Ojo OO, Okubadejo NU. An Assessment of the Knowledge and Attitudes of Graduating Medical Students in Lagos, Nigeria, Regarding Obstructive Sleep Apnea. Ann Am Thorac Soc. 2015; 12(9):1358-63.

11. Rutagarama O, Gelaye B. Risk of Common Mental Disorders in Relation to Symptoms of Obstructive Sleep Apnea Syndrome among Ethiopian College Students. J Sleep Disord Treat Care. 2015;4(3). doi: 10.4172/23259639.1000161. 